\title{
PAPER \\ Chaos-Chaos Intermittency Synchronization Induced by Feedback Signals and Stochastic Noise in Coupled Chaotic Systems
}

\author{
Sou NOBUKAWA ${ }^{\dagger a)}$, Member, Nobuhiko WAGATSUMA ${ }^{\dagger \dagger}$, Nonmember, and Haruhiko NISHIMURA ${ }^{\dagger \dagger}$, Member
}

\begin{abstract}
SUMMARY Various types of synchronization phenomena have been reported in coupled chaotic systems. In recent years, the applications of these phenomena have been advancing for utilization in sensor network systems, secure communication systems, and biomedical systems. Specifically, chaos-chaos intermittency (CCI) synchronization is a characterized synchronization phenomenon. Previously, we proposed a new chaos control method, termed as the "reduced region of orbit (RRO) method," to achieve CCI synchronization using external feedback signals. This method has been gathering research attention because of its ability to induce CCI synchronization; this can be achieved even if internal system parameters cannot be adjusted by external factors. Further, additive stochastic noise is known to have a similar effect. The objective of this study was to compare the performance of the RRO method and the method that applies stochastic noise, both of which are capable of inducing CCI synchronization. The results showed that even though CCI synchronization can be realized using both control methods under the induced attractor merging condition, the RRO method possesses higher adoptability and accomplishes a higher degree of CCI synchronization compared to additive stochastic noise. This advantage might facilitate the application of synchronization in coupled chaotic systems.
\end{abstract}

key words: chaos, synchronization, control, reduced region of orbit method

\section{Introduction}

Various kinds of synchronization phenomena have been widely observed in nonlinear systems, which are typified by social systems, electrical circuits, and biological systems (review in [1]). Among these synchronization phenomena, fluctuation enhances the degree of synchronization in stochastic resonance and chaotic resonance (review in [2], [3]). In stochastic resonance, the timing for exceeding the barrier or threshold of nonlinear systems synchronizes with a weak external signal under the effect of additive stochastic noise [4]-[9]. Stochastic resonance was first observed in climatic systems as the mechanism of the Milankovitch cycle [4]. In recent years, stochastic resonance has been observed in numerous types of systems including economic and biological systems [5]-[11]. In chaotic resonance, chaos causes a phenomenon similar to stochastic resonance [2], [12]-[17]. Chaotic resonance was initially observed in systems with chaos-chaos intermittency (CCI), in which a chaotic orbit

Manuscript received November 22, 2019.

Manuscript revised March 20, 2020.

$\dagger$ The author is with Department of Computer Science, Chiba Institute of Technology, Narashino-shi, 275-0016 Japan.

${ }^{\dagger}$ The author is with Faculty of Science, Department of Information Science, Toho University, Funabashi-shi, 274-8510 Japan.

${ }^{\dagger \dagger}$ The author is with Graduate School of Applied Informatics, University of Hyogo, Kobe-shi, 650-0047 Japan.

a)E-mail: nobukawa@cs.it-chiba.ac.jp

DOI: $10.1587 /$ transfun.2019EAP1160 hopped among separated attractor regions such as cubic maps and Chua's circuits [2], [12]-[15]. In chaotic resonance, the switching of an orbit among regions in CCI synchronizes with weak external signals; the degree of synchronization is maximized in the vicinity of attractor merging bifurcation [2]. Subsequently, the study of chaotic resonance proceeded to neural systems with CCI, such as neural systems composed of excitatory and inhibitory neurons [18], and associative memory embedded with a chaotic neural network [19], [20]. Moreover, chaotic resonance was studied for additional physiological neural systems such as cerebellar learning systems [16], [21], [22] and spiking neural systems [17], [23].

The typical applications of these synchronization phenomena include the wide utilization of the mechanism of stochastic resonance for telecommunications systems and biomedical applications [24]-[27]. In the field of telecommunications systems, Tadokoro et al. applied the mechanism of stochastic resonance to a BPSK receiver. This enabled the receiver to improve its bit error rate performance at an appropriate noise strength [27]. They also proposed a method for enhancing the sensitivity of a wireless signal for node positioning in a wireless sensor network [26]. In the field of biomedical applications, Kurita et al. developed a wearable device to enhance the tactile sensitivity of a surgeon's hands by applying appropriate vibration [24], [25]. Enders et al. and Seo et al. utilized this method to realize stochastic resonance in human sensory systems by applying vibrotactile noise and proposed a method for improving haptic sensations and a rehabilitation method for paralyzed patients [28], [29]. However, the applications of chaotic resonance have not been reported thus far, even though chaotic resonance was reported to have higher sensitivity compared to stochastic resonance [19]. A plausible reason for this is that it is necessary to adjust a chaotic state appropriately for synchronization in chaotic resonance by adjusting internal system parameters. However, this adjustment is considerably difficult in numerous cases, particularly biological systems. To find a solution to this problem, we previously proposed a chaos control method named the "reduced region of orbit (RRO) method" [30]. The RRO method enables the condition for attractor merging bifurcation to be adjusted by increasing/decreasing the local maximum/minimum values of a map function through external feedback signals. Therefore, this method optimizes the chaotic state for synchronization, and it has been applied to a cubic map [30], neural systems composed of excitatory and inhibitory neurons [31], [32], and Chua's circuit [33]. Therefore, the RRO method has the 
potential to accelerate the application of chaotic resonance.

In the synchronization among coupled chaotic oscillators, various types of synchronization phenomena have been reported, such as chaos synchronization and phase synchronization (review in [1]). The applications of chaos synchronization have been progressing to sensor network systems and secure communication systems [34]-[36]. Moreover, the phenomenon of chaotic itinerancy is known in coupled chaotic systems. Here, the transition between several regions of an attractor occurs through dynamical switching between chaotic synchronized and desynchronized states [37]. Park et al. developed a nonlinear oscillator network based on the Bonhoeffer-van der Pol model to generate the movement patterns of snake-like robots [38]. However, these applications are restricted to cases in which internal system parameters can be controlled. In contrast, the RRO method can be applied even in situations where it is difficult to control internal system parameters. Previously, our application of the RRO method to a system of coupled cubic maps confirmed that CCI synchronization occurs close to attractor merging bifurcation [39]. Additive stochastic noise is known to have the effect of inducing attractor merging bifurcation [2], [32]. Therefore, the application of additive stochastic noise is another suitable method to induce CCI synchronization. However, CCI synchronization induced by additive stochastic noise has not been evaluated in coupled nonlinear oscillators, and a comparison with CCI synchronization induced by RRO method is required to determine which method is more suitable.

In our previous study, the RRO method realized a higher sensitivity and degree of synchronization than the method that applies additive stochastic noise in the case of CCI synchronization against a weak input signal in a one-dimensional chaotic system [32]. Therefore, we hypothesized that the RRO method has higher sensitivity than the additive noise method even in CCI synchronization among nonlinear oscillators, which can be treated as higher-dimensional chaotic systems. To prove this hypothesis, the present study compared the synchronization performances of the RRO method and the method that applies additive stochastic noise, without controlling internal system parameters, as methods of inducing CCI synchronization in coupled chaotic systems. For this purpose, we evaluated the CCI synchronization in coupled cubic maps with RRO feedback signals and with the application of stochastic noise. The topology of the coupled cubic map networks was selected to include realistic network structures; hence, a ring-type network, a small-world network, and a random network were adopted.

\section{Material and Methods}

2.1 Cubic Map System with External Feedback Signals and Additive Noise

A discrete cubic map is a simple chaotic system in which CCI occurs. A cubic map with RRO feedback $K u(x)$ and Gaussian white noise $D \xi(t)$ is defined as follows:

$$
\begin{aligned}
& x(t+1)=F(x(t))+K u(x(t))+D \xi(t), \\
& F(x)=\left(a x-x^{3}\right) \exp \left(-x^{2} / b\right), \\
& u(x)=-\left(x-x_{d}\right) \exp \left(-\left(x-x_{d}\right)^{2} /\left(2 \sigma^{2}\right)\right) .
\end{aligned}
$$

Here, $D, K$, and $x_{d}$ are the strength of the noise (the mean and standard deviation of $\xi(t)$ are zero and 1.0, respectively), the strength of the feedback control, and the point dividing each attractor, respectively. In this study, we set $x_{d}=0$ because the cubic map of Eq. (2) consists of two symmetric attractor regions (i.e., positive and negative $x(t)$ regions). In the absence of external noise $(D=0)$ and an external feedback signal $(K=0)$, CCI, in which the chaotic orbit of $x(t)$ hops between the positive and negative regions of $x(t)$, arises under the condition $F\left(f_{\max }\right)<0, F\left(f_{\min }\right)>0$ [30]. Here, $f_{\max , \min }$ indicates the local maximum of the minimum of a map function. Internal parameter $a$ is set as $a=2.81,2.82$, and 2.83 , and the chaotic attractor of $x(t)$ is divided into separate positive and negative regions under conditions free from an external signals and noise. Figure 1(a), which serves as an example of the dynamics of $x(t)$, shows the map function, its orbit, and the time series of $x(t)$ at $a=2.83$.

Under the condition of $F\left(f_{\max }\right)>0, F\left(f_{\min }\right)<0$, where CCI does not occur, an external feedback signal with negative feedback strength $(K<0)$ induces the effect of increasing the absolute values of $f_{\text {max,min }}$ CCI occurs for sufficiently large values of $f_{\text {max,min }}$ that satisfy $F\left(f_{\max }\right)+K u\left(f_{\max }\right)<0, F\left(f_{\min }\right)+K u\left(f_{\min }\right)>0$ [30]. In this study, the other parameter of the RRO feedback signal was set as $\sigma=0.6$. Figure 1(b) shows the map function, its orbit, and the time series of $x(t)$ at $a=2.83$ to demonstrate the effect of RRO feedback signals with negative strength $(K=-0.3)$ under the noise-free condition $(D=0)$.

Even under the condition $F\left(f_{\max }\right)>0, F\left(f_{\min }\right)<0$ without RRO feedback signals $(K=0)$, additive stochastic noise can induce CCI [32]. A typical example of CCI induced by stochastic noise $(D=0.03)$ is shown in Fig. 1(c) for $a=2.83$.

2.2 Coupled Cubic Map System with External Feedback Signals and Additive Noise

We used a ring-type network consisting of $N$ cubic maps, where the respective cubic maps were joined by gap junctions with strength $J$ :

$$
\begin{aligned}
& x_{i}(t+1)=F\left(x_{i}(t)\right)+K u\left(x_{i}(t)\right)+D \xi(t) \\
& +J\left(2 M x_{i}-\sum_{j=i-M, j \neq i}^{j=i+M} x_{j}\right) \quad(i=1, \cdots, N) .
\end{aligned}
$$

Here, $2 M$ indicates the mean degree of a node, and the periodic boundary condition is applied at the beginning and end of a network. In this study, $M$ was set as [0.2N], where [.] indicates a Gaussian symbol. In Eq. (4), if the ordinal diffusive coupling is set such that $\sum_{j=i-M, j \neq i}^{j=i+M} x_{j}-2 M x_{i}$, according 
(a)
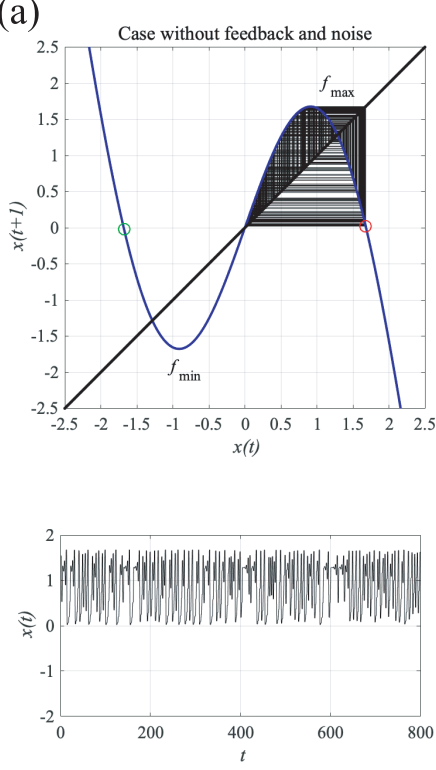

(b)
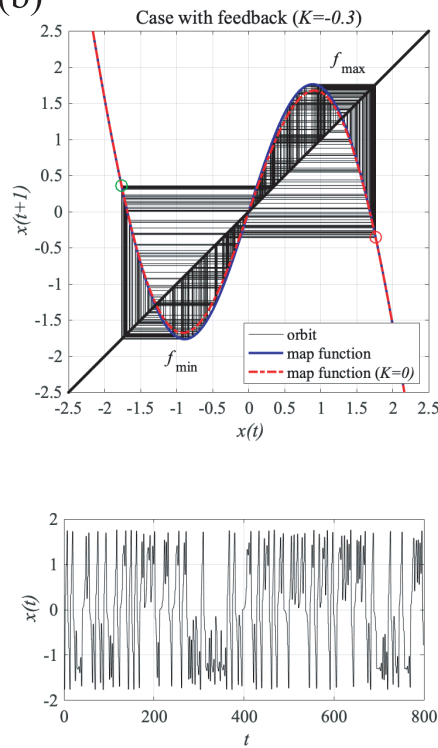

(c)
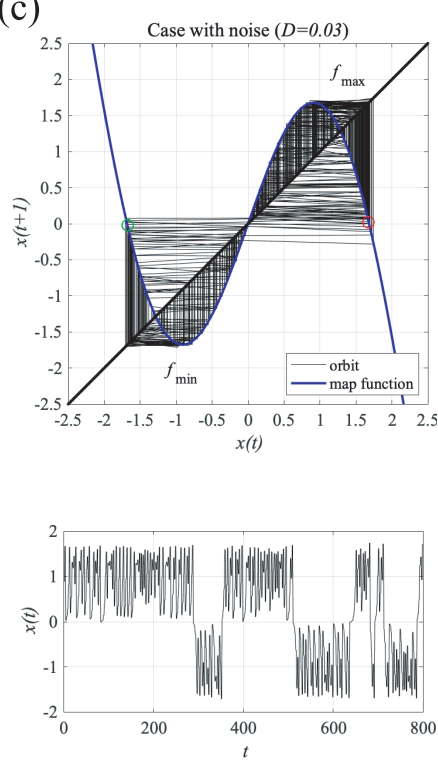

Fig. 1 Effect of attractor merging induced by reduced region of orbit (RRO) feedback and additive stochastic noise. (a) The map function of $F(x)$ (blue solid line) and orbit $x(t)$ (black solid line) (upper part) and the time series of $x(t)$ (lower part) in the case without RRO feedback signals or additive stochastic noise ( $K=0, D=0)$ at $a=2.83$. The orbit is confined to either the positive or negative $x(t)$ region, depending on initial value $x(0)$. The red and green circles indicate the values of $F\left(f_{\max }\right)>0$ and $F\left(f_{\min }\right)<0$, respectively, i.e., the condition of separated attractors is satisfied. (b) The map function of $F(x)+K u(x)$ and orbit $x(t)$ (upper part) and the time series of $x(t)$ (lower part) in the case of RRO feedback signals with negative feedback strength $(K=-0.3)$ under the noise-free condition $(D=0)$. The orbit exhibits chaos-chaos intermittency $(\mathrm{CCI})$, where the orbit hops between positive and negative $x(t)$ regions. The red and green circles indicate the values of $F\left(f_{\max }\right)+K u\left(f_{\max }\right)<0$ and $F\left(f_{\min }\right)+K u\left(f_{\min }\right)>0$, respectively, i.e., the condition of CCI is satisfied. (c) The map function of $F(x)$ and orbit $x(t)$ (upper row) and the time series of $x(t)$ (lower row) when using additive stochastic noise $(D=0.03, K=0)$. Even though the condition under which CCI arises does not occur $\left(F\left(f_{\max }\right)>0\right.$ and $\left.F\left(f_{\min }\right)<0\right)$, the additive stochastic noise induces CCI.

to the structure of the cubic map, the opposite-phase synchronization of CCI occurs at sufficient coupling strength. In this study, we used the inverse sign of diffusive coupling to evaluate synchronization with the coordinate phase [39].

In addition to ring-type networks, a small-world network and a random network were evaluated. These networks are produced by the Watts-Strogatz model [40]. Practically, the gap junctions are randomly rewired from the above ringtype network according to rewiring probability $\beta$. In this study, we used the ring-type network $(\beta=0)$, small-world network $(\beta=0.3)$, and random network $(\beta=1.0)$.

\subsection{Evaluation Index}

We evaluated the synchronization of CCI by determining the correlation coefficient between $X_{i}(t)$ and $X_{j}(t)(i, j=$ $1,2, \cdots, N, i \neq j)$. Here, $X_{i, j}$ denotes the binarized time series of $x_{i}(t)$ and $x_{j}(t)$ such that $X_{i, j}(t)=1$ in the case of $x_{i, j}(t) \geq 0$, whereas $X_{i, j}(t)=-1$ in the case of $x_{i, j}(t)<0$. The correlation coefficient of $X_{i}(t)$ and $X_{j}(t)$ is defined as follows:

$$
\begin{aligned}
& C(\tau)=\frac{C_{i j}(\tau)}{\sqrt{C_{i i} C_{j j}}} \\
& C_{i j}(\tau)=\left\langle\left(X_{i}(t+\tau)-\left\langle X_{i}\right\rangle\right)\left(X_{j}(t)-\left\langle X_{j}\right\rangle\right)\right\rangle
\end{aligned}
$$

$$
\begin{aligned}
& C_{i i}=\left\langle\left(X_{i}(t)-\left\langle X_{i}\right\rangle\right)^{2}\right\rangle \\
& C_{j j}=\left\langle\left(X_{j}(t)-\left\langle X_{j}\right\rangle\right)^{2}\right\rangle,
\end{aligned}
$$

where $\langle\cdot\rangle$ indicates temporal average $t$.

Synchronization stability was quantified by utilizing the maximum transverse Lyapunov exponent [1]. This evaluation was conducted using the method developed by Dabrowski [41]. According to Dabrowski's method, the temporal evolution of the perturbation vector, $\mathbf{d}_{\mathbf{s}}{ }^{k}\left(t_{\perp}\right)(k=$ $1,2, \cdots, M)$, from the manifold corresponding to complete synchronization during $t_{\perp} \in\left[0: \tau_{\perp}\right]$ is calculated, where the initial perturbation, $\mathbf{d}_{\mathbf{s}}{ }^{k}(0)$, is applied at $t=t_{0}+(k-1) \tau_{\perp}$. The inner product between the perturbation vector, $\mathbf{d}_{\mathbf{s}}{ }^{k}\left(\tau_{\perp}\right)$, and its temporal derivative is given by

$$
\lambda_{s}^{k}=\frac{\mathbf{d}_{\mathbf{s}}{ }^{k}\left(\tau_{\perp}\right) \cdot \frac{d \mathbf{d}_{\mathbf{s}}{ }^{k}\left(\tau_{\perp}\right)}{d t}}{\left|\mathbf{d}_{\mathbf{s}}{ }^{k}\left(\tau_{\perp}\right)\right|^{2}} .
$$

Here, $\cdot$ denotes the inner product. The maximum transverse Lyapunov exponent is defined as follows:

$$
\lambda_{\perp}=\frac{1}{\tau_{\perp} M} \sum_{k=1}^{M} \lambda_{s}^{k} .
$$

Here, we set $\tau_{\perp}=5$ and $\left|\mathbf{d}_{\mathbf{s}}{ }^{k}(0)\right|=10^{-6}$, and $\lambda_{\perp}>0$ 
indicates the state of instability of synchronization among $x_{i}(t)$.

\section{Results}

We evaluated the CCI synchronization in the coupled cubic map $(N=20)$. Each of the upper rows in Fig. 2 shows the correlation coefficient of the binarized time series of $x(t)$ : $C(\tau=0)$ as a function of RRO feedback strength $K$ and the strength of the gap junction, $J$, under the noise-free condition $(D=0)$. The results indicate that CCI does not occur in small- $|K|$ regions, which correspond to the white regions in the figure. As the value of $|K|$ increases, attractor merging bifurcation occurs, with $\max _{\tau} C(\tau)$ exhibiting a peak in the vicinity of attractor merging bifurcation. This tendency is confirmed for all network topologies (ring, small-world, and random networks). That is, RRO feedback induces attractor merging bifurcation, and the CCI synchronization exhibits a peak in the vicinity of this bifurcation. The lower rows of Fig. 2 show $C(\tau=0)$ as a function of noise strength $D$ and the strength of the gap junction, $J$, without RRO feedback $(K=0)$. CCI does not occur in small- $D$ regions, which correspond to the white regions in the figure. An increase in the value of $D$ causes attractor merging bifurcation, with $C(\tau=0)$ exhibiting a peak in the vicinity of attractor merging bifurcation. This tendency is also confirmed for all network topologies. That is, in addition to the RRO feedback strength, the additive stochastic noise induces attractor merging bifurcation, and the CCI synchronization exhibits a peak close to this bifurcation. A typical time series of $x_{i}(t)$ is shown in Fig. 3(a) for a small-world network jointed by a gap junction $\left(J=3.0 \times 10^{-3}\right)$ corresponding to that shown in Fig. 2(b) with $a=2.82$. This result shows that the RRO feedback signal and additive stochastic noise induce CCI synchronization $(C(\tau=0) \approx 0.65)$. On the other hand, Fig. 3(b) shows the time series of $x_{i}(t)$ in the cases where $K$ and the noise strength $D$ have larger absolute values than the appropriate ones shown in Fig.3(a). Owing to the effect of a larger RRO feedback signal or additive stochastic noise, the CCI frequency becomes high; consequently, CCI synchronization does not occur $(C(\tau=0) \lesssim 0.1)$.

In addition to CCI synchronization, we investigated the synchronization stability of $x_{i}(t)$ in the coupled cubic maps. In the parameter regions corresponding to Fig. 2, the transverse Lyapunov exponent, $\lambda_{\perp}$, exhibits positive values, $\lambda_{\perp} \approx 0.15$, with the RRO feedback signal and additive stochastic noise. Therefore, the instability of the synchronization of $x_{i}(t)\left(\lambda_{\perp}>0\right)$ is maintained even if CCI synchronization is achieved.

CCI synchronization was additionally evaluated for a larger network. Figure 4 shows the correlation coefficient, $C(\tau=0)$, as a function of network size $N$ in the case of CCI synchronization induced by RRO feedback signals and additive stochastic noise. In the case of RRO feedback signals, $K$ is set to achieve the maximum $C(\tau=0)$ in the range of $-0.05 \leq K \leq 0$. In the case of additive stochastic noise, $D$ is set to achieve the maximum $C(\tau=0)$ in the range of
$0 \leq D \leq 0.025$. Here, two kinds of gap junctions were used, $J=3.0 \times 10^{-3}$ and $5.0 \times 10^{-4}$, and the internal parameter $a$ was set as $a=2.82$. These results show that CCI synchronization decreases as $N$ increases. In the weaker gap junction $\left(J=5.0 \times 10^{-4}\right)$, the degree of CCI synchronization induced by the RRO feedback signals is higher than that induced by stochastic noise; this tendency is particularly significant for small networks with $N \lesssim 80$.

\section{Discussion and Conclusion}

In this study, we controlled CCI synchronization in a coupled cubic map system by adjusting the negative strength of RRO feedback and by utilizing additive stochastic noise. As the index of this synchronization, we utilized the transverse Lyapunov exponent and the correlation of the timing of CCI among coupled cubic maps. The results indicated that the degree of CCI synchronization reaches a maximum in the vicinity of attractor merging bifurcation, thereby maintaining the synchronization instability among orbits in both cases. These tendencies are preserved for the different internal parameter settings and network topologies. However, CCI synchronization degrades as network size increases. Moreover, a comparison between the CCI synchronization induced by the RRO feedback signal and that induced by additive stochastic noise revealed that the former achieves a higher degree of synchronization.

First, the reason why CCI synchronization reaches a maximum in the vicinity of attractor merging bifurcation must be considered. Close to the attractor merging bifurcation point, autonomous CCI seldom occurs when coupling does not occur. Under this condition, the perturbation caused by the CCI from other oscillators induces CCI in the coupled network. This interaction achieves a high degree of synchronization. This tendency is congruent with the case of positive RRO feedback strength in our previous study [39]. In addition, the degree of synchronization of CCI against a weak external signal was reported to exhibit a peak close to attractor merging bifurcation in systems with CCI [2], [12]-[15], [30]-[32]. Moreover, our previous studies demonstrated that the degree of synchronization induced by adjusting the order parameter to control attractor merging bifurcation was higher than that induced by stochastic noise; this tendency was particularly significant for a weak input signal [19], [32]. The results obtained in this study are congruent with this finding.

It is also necessary to compare the RRO feedback method with conventional chaos control methods [42]-[45] and nonlinear control methods for synchronization [46][49]. Conventional chaos control methods, such as the Ott-Grebogi-Yorke method and $H_{\infty}$ method [42]-[45], have been used to remove chaotic behavior and to reach an equilibrium state or a stable periodic state. However, the RRO feedback method does not remove the chaotic state and instead achieves a transition to the appropriate chaotic state for synchronization. Nonlinear control methods have been proposed for achieving chaos synchronization; these meth- 
(a)
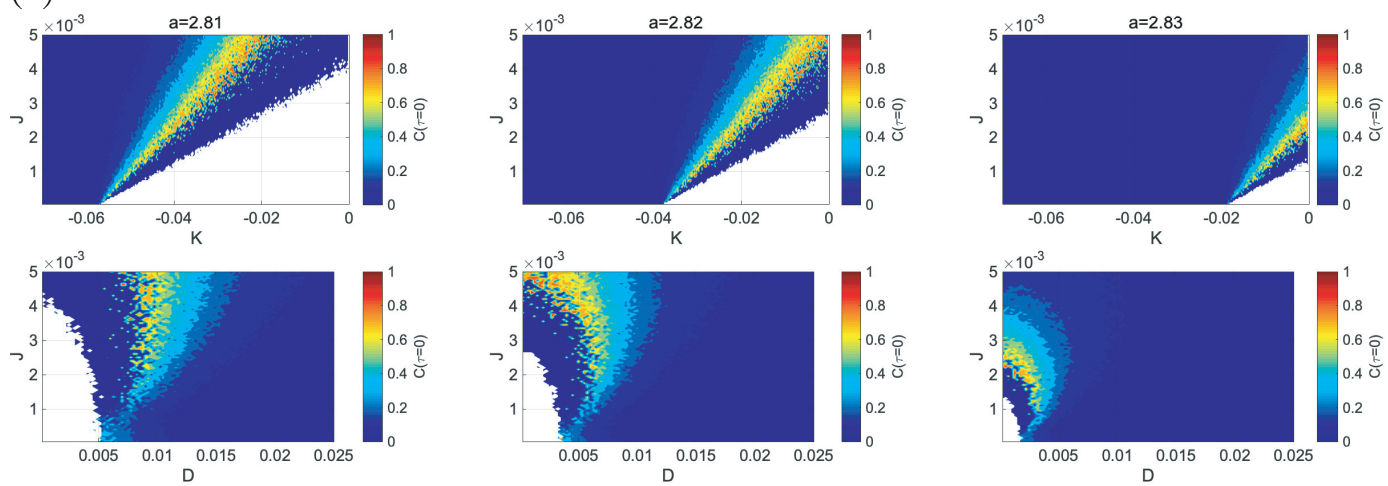

(b)
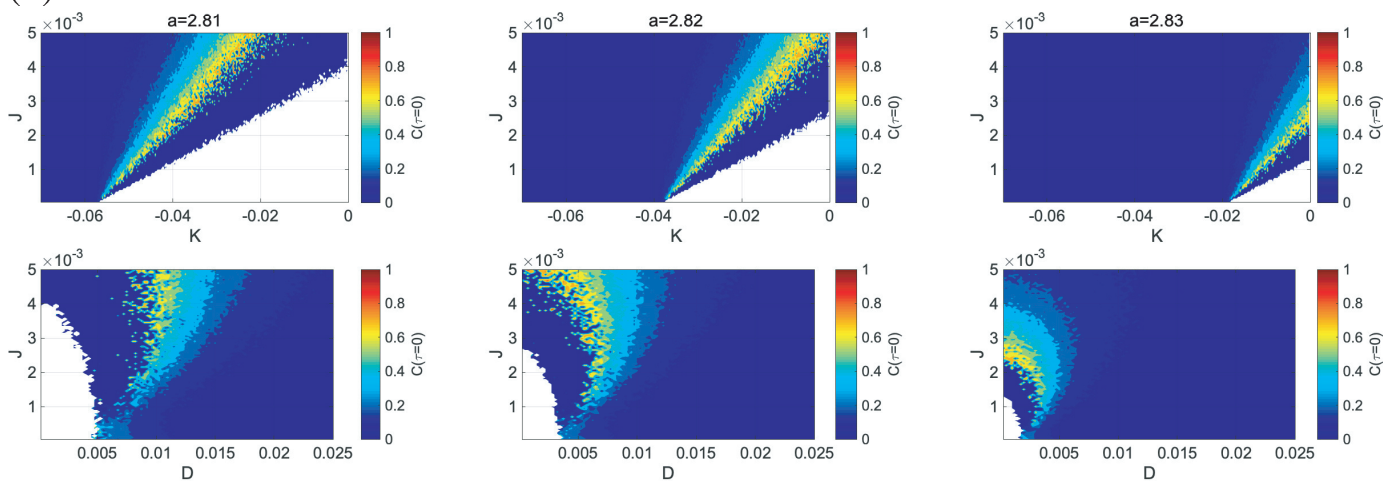

(c)
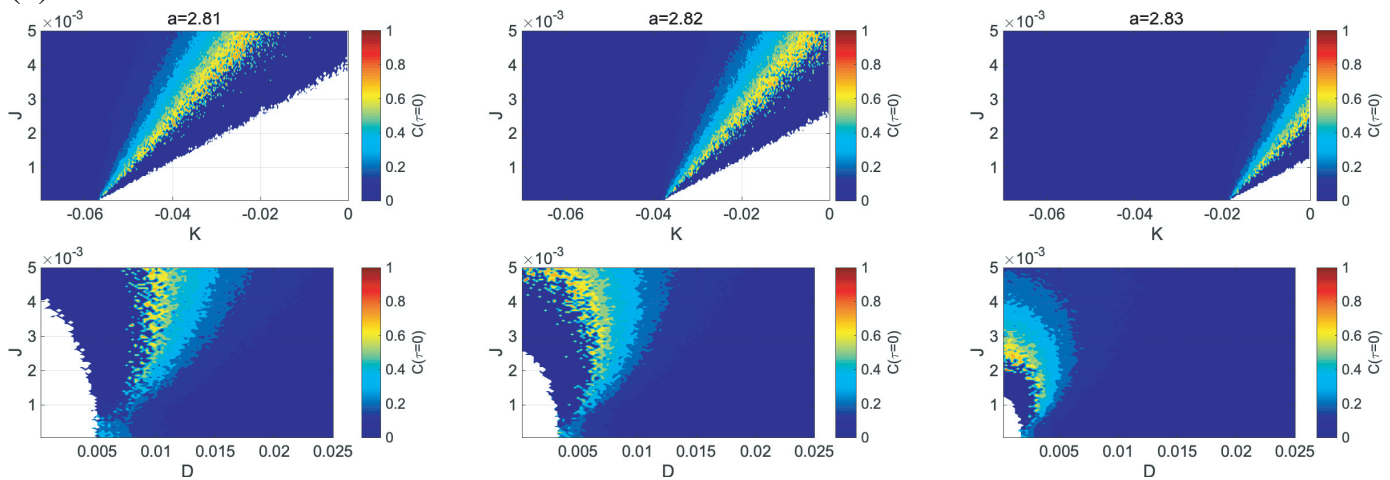

Fig. 2 CCI synchronization in the coupled cubic maps $(N=20)$. The correlation coefficient of the binarized time series of $x(t): C(\tau=0)$ as a function of RRO feedback strength $K$ and the strength of the gap junction, $J$, in the noise-free condition $(D=0)$ (upper row). Correlation coefficient $C(\tau=0)$ as a function of the strength of additive noise $D$ and strength of the gap junction, $J$, (lower row) in the absence of RRO feedback signals $(K=0)$. (a) Ring network example corresponding to rewiring probability $\beta=0$. (b) Small-world network example corresponding to $\beta=0.3$. (c) Random network example corresponding to $\beta=1.0$. The RRO feedback strength and the additive stochastic noise induce attractor merging bifurcation, and the CCI synchronization exhibits a peak close to this bifurcation.

ods utilize Lyapunov stability, active control, and converse Lyapunov theories [46]-[49]. In contrast to these methods, the RRO feedback method maintains the instability of synchronization among nonlinear oscillators. In recent years, the co-existing states of synchronization and asynchronization, which are typified by chaotic itinerancy [50] and the Chimera state [51], have been utilized to generate the moving patterns of snake-like robots, instead of using complete synchronization [38], [52]. The RRO feedback method can also be utilized for these applications.

Furthermore, we must discuss the adoptability of the control methods based on RRO feedback signals and additive stochastic noise. In our previous study, we evaluated the control of CCI synchronization by RRO feedback signals with positive strength in coupled cubic maps [39]. In the case of positive RRO feedback strength, the frequency of CCI decreases with increasing feedback strength, and the degree of synchronization reaches a maximum in the proxim- 
(a)
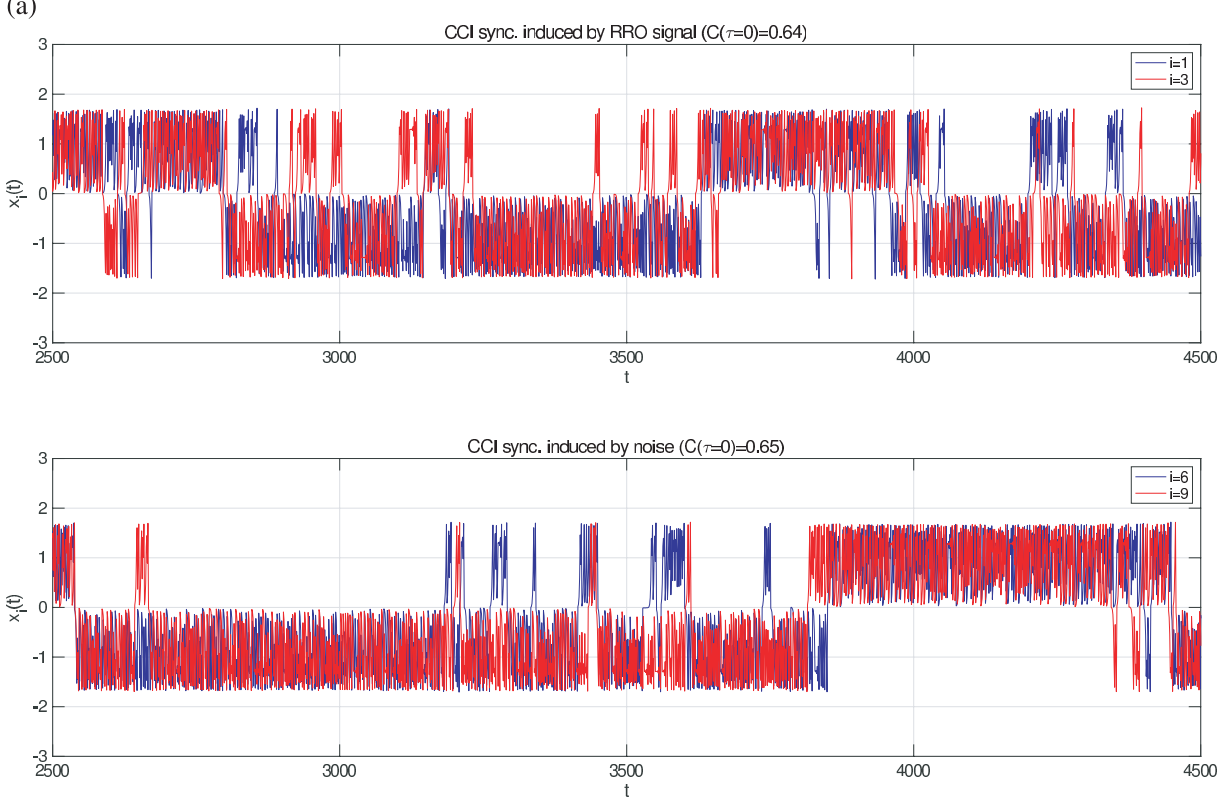

(b)
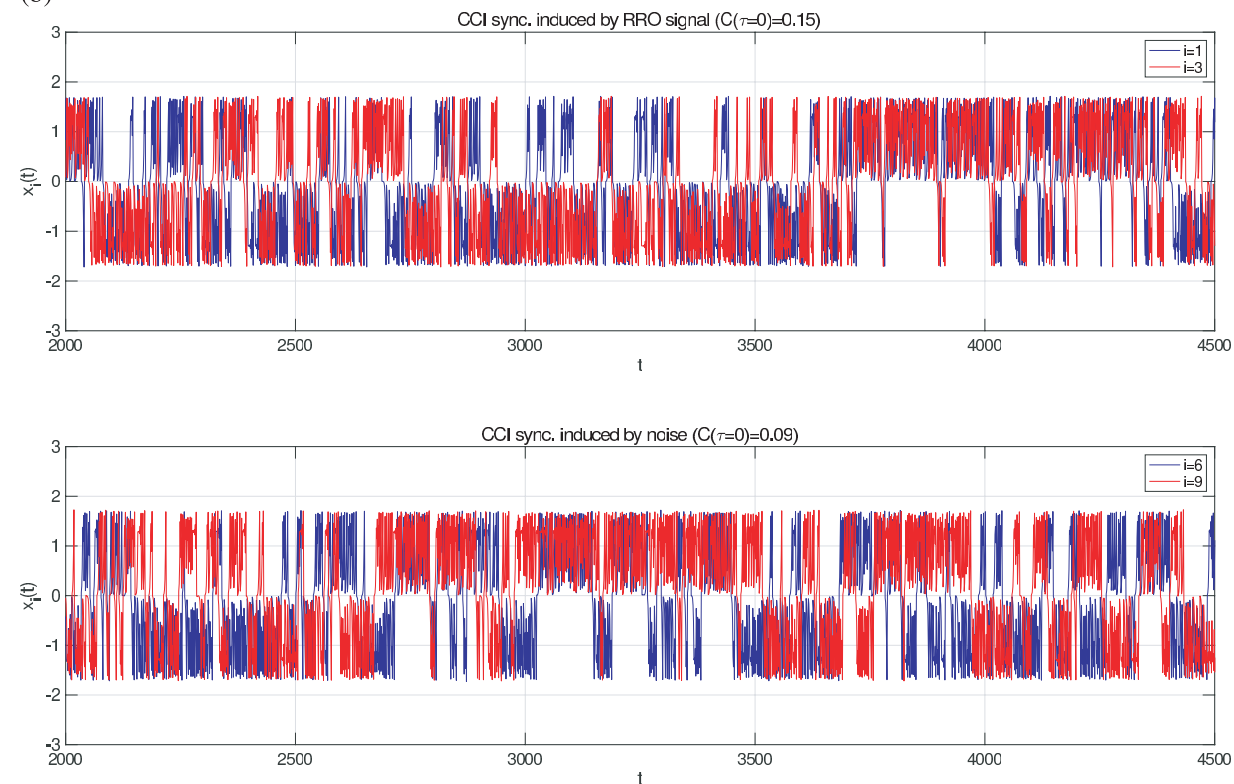

Fig. 3 Typical time series of $x_{i}(t)$ in the coupled cubic maps for a small-world network topology $(\beta=0.3)$ at $a=2.82$ and $J=3 \times 10^{-3}$. (a) Typical time series in a state for CCI synchronization. The upper and lower rows correspond to the CCI synchronization induced by the RRO feedback signal $(K=-0.02)$ and that induced by additive stochastic noise $(D=0.0075)$, respectively. (b) Typical time series of $x_{i}(t)$ when CCI synchronization does not occur. The upper and lower rows correspond to the time series with the RRO method $(K=-0.03)$ and that with the method applying additive stochastic noise $(D=0.015)$, respectively.

ity of attractor merging bifurcation. In contrast, for negative feedback strength, CCI is induced under the condition of separated attractors as the absolute value of negative feedback strength increases, and the degree of CCI exhibits a peak close to attractor merging bifurcation. In contrast to RRO feedback signals, additive stochastic noise only induces CCI and does not decrease the frequency of CCI. Therefore, the control of RRO feedback signals is more adoptable compared to additive noise.
Moreover, we must consider why the degrees of CCI synchronization exhibited almost the same profiles in their dependencies on the RRO feedback strength and noise strength among different network topologies (see Figs. 2 and 4). It is known that a random rewiring process in the WattsStrogatz model induces synchronization states [53], [54]. Therefore, the small-world network and random network generally reach the synchronization state more easily than ring networks. However, in this study, the degree of a node 

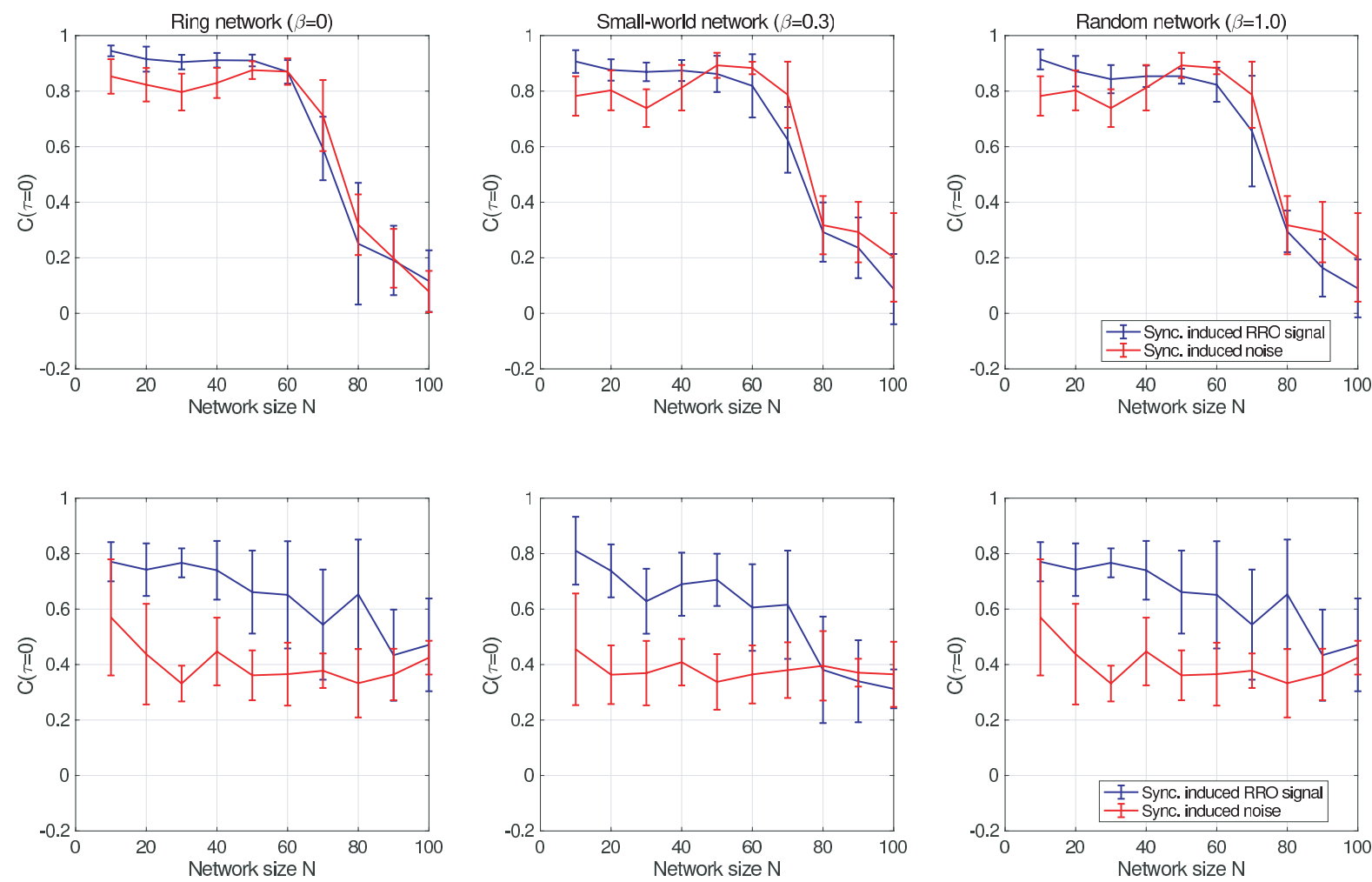

Fig. 4 Correlation coefficient $C(\tau=0)$ as a function of network size $N$ in CCI synchronization induced by RRO feedback signals and additive stochastic noise. The upper and lower rows indicate the situations with a gap junction of $J=3.0 \times 10^{-3}$ and $J=5.0 \times 10^{-4}$, respectively, where the internal parameter $a$ is set as $a=2.82$. Error bars indicate the standard deviation among ten trials.

is set to a large value (the mean degree of a node corresponds to $2 M$, where $M$ is set to $[0.2 N])$. In such a highly dense network, the effect of the rewiring process of the Watts-Strogatz model is insignificant for the synchronization state, which is why no significant difference was observed in the synchronization profiles among different network topologies. On the other hand, with a smaller degree of a node, differences among network topologies might be significant, but the parameter regions where CCI synchronization appears rigidly are restricted. To find these parameter regions, the dependence of CCI synchronization on the internal parameter, network size, noise strength, and RRO feedback strength needs to be evaluated.

A limitation of this study is that the application of the RRO feedback method to an actual nonlinear system, such as a typical biomedical application, requires the RRO feedback method to be applied to continuous nonlinear systems. For this purpose, we developed preliminary RRO methods and applied them to Chua's circuit system [33]. In the future, we plan to use this method by considering CCI synchronization in coupled continuous chaotic neural systems. Moreover, we will evaluate the CCI synchronization in networks with different topologies under a small degree of a node.

In conclusion, in this study, we evaluated the control of CCI synchronization by RRO feedback signals and additive stochastic noise in coupled cubic maps. The results confirmed that even though CCI synchronization can be re- alized using both control methods in the vicinity of induced attractor merging, the RRO feedback method exhibits higher adoptability and a higher degree of CCI synchronization compared to additive stochastic noise. The widespread application of various types of synchronization is in progress. The advantages of the RRO feedback method might facilitate these applications.

\section{References}

[1] A. Pikovsky, M. Rosenblum, and J. Kurths, Synchronization: A Universal Concept in Nonlinear Sciences, Cambridge University Press, 2003.

[2] V.S. Anishchenko, V. Astakhov, A. Neiman, T. Vadivasova, and L. Schimansky-Geier, Nonlinear Dynamics of Chaotic and Stochastic Systems: Tutorial and Modern Developments, Springer Science \& Business Media, 2007.

[3] S. Rajasekar and M.A. Sanjuan, Nonlinear Resonances, Springer, 2016.

[4] R. Benzi, A. Sutera, and A. Vulpiani, "The mechanism of stochastic resonance,” J. Phys. A: Math. Gen., vol.14, no.11, pp.L453-L457, 1981.

[5] F. Moss and K. Wiesenfeld, "The benefits of background noise," Sci. Am., vol.273, no.2, pp.66-69, 1995.

[6] L. Gammaitoni, P. Hänggi, P. Jung, and F. Marchesoni, "Stochastic resonance," Rev. Mod. Phys., vol.70, no.1, pp.223-287, 1998.

[7] P. Hänggi, "Stochastic resonance in biology how noise can enhance detection of weak signals and help improve biological information processing," ChemPhysChem, vol.3, no. 3, pp.285-290, 2002.

[8] S. Nobukawa and H. Nishimura, "Enhancement of spike-timing- 
dependent plasticity in spiking neural systems with noise," Int. J. Neur. Syst., vol.26, no.05, p.1550040, 2016.

[9] S. Nobukawa, R. Hashimoto, H. Nishimura, T. Yamanishi, and M. Chiba, "Noise-induced phenomena in the Kaldor business cycle model," Trans. Institute of Systems, Control and Information Engineers, vol.30, no.12, pp.459-466, 2017

[10] T. Mori and S. Kai, "Noise-induced entrainment and stochastic resonance in human brain waves," Phys. Rev. Lett., vol.88, no.21, p.218101, 2002.

[11] M.D. McDonnell and L.M. Ward, "The benefits of noise in neural systems: Bridging theory and experiment," Nat. Rev. Neurosci. vol.12, no.7, pp.415-426, 2011.

[12] T. Carroll and L. Pecora, "Stochastic resonance and crises," Phys. Rev. Lett., vol.70, no.5, pp.576-579, 1993.

[13] T. Carroll and L. Pecora, "Stochastic resonance as a crisis in a perioddoubled circuit," Phys. Rev. E, vol.47, no.6, pp.3941-3949, 1993.

[14] A. Crisanti, M. Falcioni, G. Paladin, and A. Vulpiani, "Stochastic resonance in deterministic chaotic systems," J. Phys. A: Math. Gen., vol.27, no.17, pp.597-603, 1994.

[15] G. Nicolis, C. Nicolis, and D. McKernan, "Stochastic resonance in chaotic dynamics," J. Stat. Phys., vol.70, no.1-2, pp.125-139, 1993.

[16] S. Nobukawa and H. Nishimura, "Chaotic resonance in coupled inferior olive neurons with the Llinás approach neuron model," Neural Comput., vol.28, no.11, pp.2505-2532, 2016.

[17] S. Nobukawa, H. Nishimura, and T. Yamanishi, "Chaotic resonance in typical routes to chaos in the Izhikevich neuron model," Sci. Rep., vol.7, no.1, p.1331, 2017.

[18] S. Sinha and B.K. Chakrabarti, "Deterministic stochastic resonance in a piecewise linear chaotic map," Phys. Rev. E, vol.58, no.6, pp.8009-8012, 1998.

[19] H. Nishimura, N. Katada, and K. Aihara, "Coherent response in a chaotic neural network," Neural Process. Lett., vol.12, no.1, pp.4958,2000 .

[20] S. Nobukawa, H. Nishimura, and T. Yamanishi, "Evaluation of chaotic resonance by Lyapunov exponent in attractor-merging type systems," International Conference on Neural Information Processing, pp.430-437, Springer, 2016.

[21] N. Schweighofer, K. Doya, H. Fukai, J.V. Chiron, T. Furukawa, and M. Kawato, "Chaos may enhance information transmission in the inferior olive," Proc. National Academy of Sciences, vol.101, no.13, pp.4655-4660, 2004.

[22] I.T. Tokuda, H. Hoang, and M. Kawato, "New insights into olivocerebellar circuits for learning from a small training sample," Curr Opin. Neurobiol., vol.46, pp.58-67, 2017.

[23] S. Nobukawa, H. Nishimura, T. Yamanishi, and J.Q. Liu, "Analysis of chaotic resonance in Izhikevich neuron model," PloS ONE, vol.10, no.9, p.e0138919, 2015.

[24] Y. Kurita, M. Shinohara, and J. Ueda, "Wearable sensorimotor enhancer for fingertip based on stochastic resonance effect," IEEE Trans. Human-Mach. Syst., vol.43, no.3, pp.333-337, 2013.

[25] Y. Kurita, Y. Sueda, T. Ishikawa, M. Hattori, H. Sawada, H. Egi, H. Ohdan, J. Ueda, and T. Tsuji, "Surgical grasping forceps with enhanced sensorimotor capability via the stochastic resonance effect," IEEE/ASME Trans. Mechatronics, vol.21, no.6, pp.26242634, 2016.

[26] D. He, "A novel wireless sensor networks multilateration positioning method based on quartic double-well bistable stochastic resonance technique," Nonlinear Theory and Its Applications, IEICE, vol.8, no.1, pp.49-57, 2017.

[27] Y. Tadokoro, H. Tanaka, Y. Nakashima, T. Yamazato, and S. Arai, "Enhancing a BPSK receiver by employing a practical parallel network with stochastic resonance," Nonlinear Theory and Its Applications, IEICE, vol.10, no.1, pp.106-114, 2019.

[28] L.R. Enders, P. Hur, M.J. Johnson, and N.J. Seo, "Remote vibrotactile noise improves light touch sensation in stroke survivors' fingertips via stochastic resonance," J. Neuroengineering and Rehabilitation, vol.10, no.1, p.105, 2013.
[29] N.J. Seo, M.L. Kosmopoulos, L.R. Enders, and P. Hur, "Effect of remote sensory noise on hand function post stroke," Front. Hum. Neurosci., vol.8, p.934, 2014.

[30] S. Nobukawa, H. Nishimura, T. Yamanishi, and H. Doho, "Controlling chaotic resonance in systems with chaos-chaos intermittency using external feedback," IEICE Trans. Fundamentals, vol.E101-A, no.11, pp.1900-1906, Nov. 2018.

[31] S. Nobukawa and N. Shibata, "Controlling chaotic resonance using external feedback signals in neural systems," Sci. Rep., vol.9, no.1, p.4990, 2019

[32] S. Nobukawa, N. Shibata, H. Nishimura, H. Doho, N. Wagatsuma, and T. Yamanishi, "Resonance phenomena controlled by external feedback signals and additive noise in neural systems," Sci. Rep., vol.9, no.1, p.12630, 2019

[33] S. Nobukawa, H. Doho, N. Shibata, H. Nishimura, and T. Yamanishi, "Chaos-chaos intermittency synchronization controlled by external feedback signals in chua's circuits," IEICE Trans. Fundamentals, vol.E103-A, no.1, pp.303-312, Jan. 2020.

[34] B. Wang, S. Zhong, and X. Dong, "On the novel chaotic secure communication scheme design," Communications in Nonlinear Science and Numerical Simulation, vol.39, pp.108-117, 2016.

[35] B. Vaseghi, M.A. Pourmina, and S. Mobayen, "Secure communication in wireless sensor networks based on chaos synchronization using adaptive sliding mode control," Nonlinear Dyn., vol.89, no.3, pp.1689-1704, 2017.

[36] B. Vaseghi, M.A. Pourmina, and S. Mobayen, "Finite-time chaos synchronization and its application in wireless sensor networks," Trans. Instit. Meas. Control, vol.40, no.13, pp.3788-3799, 2018.

[37] K. Kaneko and I. Tsuda, "Chaotic itinerancy," Chaos: An Interdisciplinary Journal of Nonlinear Science, vol.13, no.3, pp.926-936, 2003

[38] J. Park, H. Mori, Y. Okuyama, and M. Asada, "Chaotic itinerancy within the coupled dynamics between a physical body and neural oscillator networks," PloS ONE, vol.12, no.8, p.e0182518, 2017.

[39] S. Nobukawa, H. Nishimura, T. Yamanishi, and H. Doho, "Induced synchronization of chaos-chaos intermittency maintaining asynchronous state of chaotic orbits by external feedback signals," IEICE Trans. Fundamentals, vol.E102-A, no.3, pp.524-531, March 2019

[40] D.J. Watts and S.H. Strogatz, "Collective dynamics of 'small-world' networks," Nature, vol.393, no.6684, pp.440-442, 1998.

[41] A. Dabrowski, "The largest transversal Lyapunov exponent and master stability function from the perturbation vector and its derivative dot product (TLEVDP)," Nonlinear Dyn., vol.69, no.3, pp.12251235,2012

[42] E. Ott, C. Grebogi, and J.A. Yorke, "Controlling chaos," Phys. Rev Lett., vol.64, no.11, pp.1196-1199, 1990.

[43] K. Pyragas, "Continuous control of chaos by self-controlling feedback," Phys. Lett. A, vol.170, no.6, pp.421-428, 1992.

[44] H. Nakajima, "On analytical properties of delayed feedback control of chaos," Phys. Lett. A, vol.232, no.3-4, pp.207-210, 1997.

[45] W. Jiang, Q. Guo-Dong, and D. Bin, "Ho variable universe adaptive fuzzy control for chaotic system," Chaos, Solitons \& Fractals, vol.24, no.4, pp.1075-1086, 2005.

[46] L. Huang, R. Feng, and M. Wang, "Synchronization of chaotic systems via nonlinear control," Phys. Lett. A, vol.320, no.4, pp.271-275, 2004

[47] H.K. Chen, "Global chaos synchronization of new chaotic systems via nonlinear control," Chaos, Solitons \& Fractals, vol.23, no.4, pp.1245-1251, 2005.

[48] M. Yassen, "Chaos synchronization between two different chaotic systems using active control," Chaos, Solitons \& Fractals, vol.23, no.1, pp.131-140, 2005.

[49] H.H. Chen, G.J. Sheu, Y.L. Lin, and C.S. Chen, "Chaos synchronization between two different chaotic systems via nonlinear feedback control," Nonlinear Analysis: Theory, Methods \& Applications, vol.70, no.12, pp.4393-4401, 2009. 
[50] K. Kaneko and I. Tsuda, Complex Systems: Chaos and Beyond: A Constructive Approach with Applications in Life Sciences, Springer Science \& Business Media, 2011.

[51] D.M. Abrams and S.H. Strogatz, "Chimera states for coupled oscillators," Phys. Rev. Lett., vol.93, no.17, p.174102, 2004.

[52] F. Kubandt, M. Nowak, T. Koglin, C. Gros, and B. Sándor, "Embodied robots driven by self-organized environmental feedback," Adapt. Behav., p.1059712319855622, 2019.

[53] C. Li and G. Chen, "Phase synchronization in small-world networks of chaotic oscillators," Physica A: Statistical Mechanics and its Applications, vol.341, pp.73-79, 2004.

[54] H. Hong, M.Y. Choi, and B.J. Kim, "Synchronization on small-world networks," Phys. Rev. E, vol.65, no.2, p.026139, 2002.

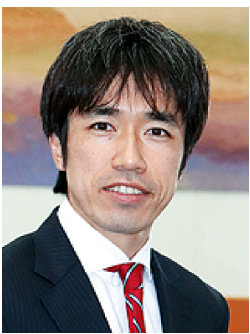

Sou Nobukawa graduated from the Department of Physics and Earth Sciences, University of Ryukyus in 2006, completed the doctoral program at University of Hyogo, and received his $\mathrm{Ph} . \mathrm{D}$. degree in 2013. He is an associate professor in the Department of Computer Science, Chiba Institute of Technology. His research interests include chaos/bifurcation and neural networks. He is a member of IEEE, INNS, IEICE, IPSJ, SICE, ISCIE, and others. He was awarded the SICE encouraging prize in 2016, the Young Researcher Award by the IEEE Computational Intelligence Society Japan Chapter, and Best Paper Award of The 29th Symposium on Fuzzy, Artificial Intelligence, Neural Networks and Computational Intelligence in 2019.

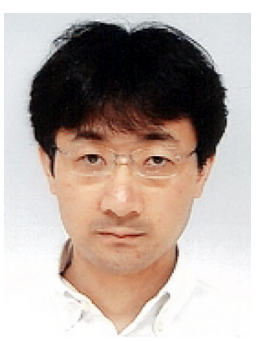

Nobuhiko Wagatsuma received his B.S., M.S., and Ph.D. degrees from University of Tsukuba in 2004, 2006, and 2009, respectively. Currently, he is a lecturer at Faculty of Science, Department of Information Science, Toho University. His research interests include visual attention and computational neuroscience.

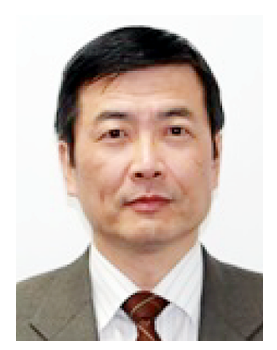

Haruhiko Nishimura graduated from the Department of Physics, Shizuoka University in 1980, completed the doctoral program at Kobe University, and received his Ph.D. degree in 1985. Currently, he is a professor in the Graduate School of Applied Informatics, University of Hyogo. His research interests include intelligent systems science based on several architectures such as neural networks and complex systems. In addition, he is presently engaged in research in biomedical, healthcare, and high confidence sciences. He is a member of the IEEE, IEICE, IPSJ, ISCIE, JNNS, and others. He was awarded the ISCIE paper prize in 2001 and the JSKE paper prize in 2010. 